\title{
A CONSTRUÇÃO DA IDENTIDADE CULTURAL DO PROFESSOR DE LÍNGUA INGLESA EM FORMAÇÃO INICIAL
}

RESUMO: Este estudo apresenta parte dos resultados da pesquisa de mestrado - situada na Linha de Pesquisa Linguagens e Educação do Programa de Pós-Graduação em Letras: Cultura, Educação e Linguagens -, que analisou como se dá a construção da identidade do professor de língua inglesa (LI) em formação inicial em tempos de interculturalidade. Este trabalho é filiado aos estudos da Linguística Aplicada, em diálogo com vozes como as de Rajagopalan (2003, 2009), Lima (2009), entre outros. No entanto, buscamos apoio em autores de outras áreas, como Bakhtin (2011), Estermann (2010), Hall (2005), Bauman (2005) e Thompson (1995). Trata-se de um estudo de caso, com natureza etnográfica, tendo como objetivo geral analisar como a construção da identidade cultural do professor de língua inglesa, em formação inicial, vem sendo construída dentro da universidade. Assim, os objetivos específicos são: explorar a constituição da identidade cultural do professor de línguas em formação inicial; identificar possibilidades de investigação que possam contribuir para refletir sobre a construção da identidade cultural do professor de LI. Os participantes da pesquisa são quatro professores em formação inicial no Curso de Letras Língua Inglesa e Literaturas de uma Universidade situada no Alto Sertão Baiano. Desse modo, com base nas análises das entrevistas dos sujeitos da pesquisa, os resultados encontrados mostraram que a construção da identidade cultural do professor de LI em formação inicial se dá por intermédio das características do mundo globalizado.

PALAVRAS-CHAVE: Cultura; Formação inicial de professor de inglês; Identidade.

\footnotetext{
* Mestre em Letras pela Universidade Estadual do Sudoeste da Bahia (Uesb).

** Doutor em Educação/Estudos da Linguagem pela Southern Illinois University (EUA). Professor Pleno da Universidade Estadual do Sudoeste da Bahia (Uesb).
} 


\section{Palavras Iniciais}

A língua inglesa não ocupa o posto de principal língua de comunicação na atualidade de forma aleatória. Muitos fatores contribuíram para esse efeito; vários deles são atribuídos à força do poderio econômico da Inglaterra no período da Revolução Industrial, bem como ao fato de os Estados Unidos (EUA) se tornarem a maior potência mundial após sair "vencedor" da Segunda Guerra Mundial, tendo em vista que as forças econômicas e militares daquele país foram os principais aliados deste último, na época. Conforme atesta Crystal (2003, p. 120, tradução nossa), "nos séculos XVII e XVIII, o inglês foi a língua da nação líder da colonização - a Grã-Bretanha. Já [...] no final do século XIX e início do século XX, ele foi o idioma do principal poder econômico - os EUA ${ }^{1}$."

Nesse contexto, para adquirir o status de língua mundial, o inglês percorreu o processo histórico de colonização da Inglaterra, principalmente sobre os Estados Unidos, visto que, com o passar do tempo, o colonizado saiu da condição de colônia, no caso dos EUA, para assumir o legado de principal potência mundial.

Nesse sentido, ao apresentarmos alguns dos fatores que transformaram a língua inglesa em idioma da comunicação global, assim, propomos neste estudo uma investigação a partir de um estudo de caso, sob os paradigmas qualitativo e etnográfico, para responder à problemática: como se dá a construção da identidade cultural do professor de língua inglesa (LI) em formação inicial? Para tanto, a base teórica centra-se na Linguística Aplicada (doravante LA), que se estabelece partindo do objetivo de alavancar a qualidade do ensino/aprendizagem de línguas - no nosso caso, a língua inglesa. Dessa forma, a LA se posiciona a partir de uma abordagem crítica, que leva em consideração o uso real da linguagem por sujeitos ideologicamente marcados, o que objetiva o foco na língua em uso. Conforme destaca Signorini (2006, p. 186), isso exige "uma compreensão aprofundada de sua dimensão histórica, social e política". Trata-se, portanto, de perceber quais enunciados

\footnotetext{
${ }^{1}$ In the seventeenth and eighteen centuries English was the language of the leading colonial nation - Britain [...]. In the late nineteenth and the early twentieth it was the language of the leading economic power - the USA.
} 
são determinantes para a construção da identidade do professor de LI em formação inicial nos deslocamentos enunciativos, demarcados historicamente na sociedade e no ambiente escolar, provocados pela globalização.

Frente a isso, conforme argumenta Bakhtin (2002, p. 132), "a cada palavra da enunciação que estamos em processo de compreender, fazemos corresponder uma série de palavras nossas". Dito de outro modo, todo enunciado remete a outro enunciado que já foi dito e mostrado em outro momento historicamente situado, mediante outros valores culturais contextualizados.

Dado ao exposto, os objetivos propostos para responder à problemática deste estudo são, a saber: objetivo geral: analisar o processo de construção da identidade cultural do professor de língua inglesa em formação inicial, a partir de um estudo de caso, com quatro futuros professores de LI, cursando o VIII semestre do curso de Licenciatura em língua inglesa e Literaturas, em uma Universidade situada no Estado da Bahia. Já os objetivos específicos são: explorar a constituição da identidade do professor de LI em formação inicial, para entender o quanto a língua está atravessada por questões políticas/ideológicas e identificar possibilidades de investigação que visem contribuir para reflexão acerca da constituição da identidade cultural do professor de LI em formação inicial.

Vale ressaltar que este texto apresenta parte dos resultados da pesquisa de mestrado filiada ao Programa de Pós-Graduação em Letras: Cultura, Educação e Linguagens -PPGCEL- UESB de Vitória da Conquista, Bahia. O estudo vincula-se na Linha de Pesquisa Linguagens e Educação do Programa citado.

Esta pesquisa foi submetida ao Comitê de Ética e Pesquisa (CEP) da Universidade Estadual do Sudoeste da Bahia (UESB). Primeiro, foram apresentados aos sujeitos da pesquisa os percursos legais e éticos exigidos pelo CEP, como a coleta das assinaturas deles nas declarações e nos termos de consentimentos. Em seguida, depois de colher todas as assinaturas legais dos participantes, o projeto de pesquisa foi submetido ao CEP, sendo aprovado com o Protocolo do Comitê de Pesquisa/Aprovação no 2.421.938. Assim, tendo a liberação do Comitê, a pesquisa foi iniciada. 
Uma vez exposta algumas considerações iniciais, passemos, a seguir, para seção de embasamento teórico, a fim de apresentarmos os autores pilares da pesquisa.

\section{Escopo Teórico}

O escopo teórico deste estudo é enunciado a partir dos pilares da Linguística Aplicada, com destaque, no Brasil, para estudiosos como Rajagopalan (2003; 2009) e Lima (2009), dentre outros. Além disso, no decorrer do texto, movimentamos conceitos de identidades, visto que as noções de autores como Bauman (2005) e Hall (2005) são muito importantes para alcançar o objetivo geral.

Nesse contexto, a escolha teórica justifica-se pela possibilidade de investigar e interpretar os sujeitos nas suas práticas sociais, as quais permitem-lhes a manifestação da linguagem, estando inseridos na história, uma vez que a construção da identidade do professor de língua inglesa em formação inicial está sendo construída no contexto da interação entre culturas, no contexto das manifestações históricas instauradas pelo processo de globalização.

Diante disso, faz-se necessário dissertar sobre globalização, que, segundo Bauman (1999, p. 7), “é o destino irremediável do mundo, um processo irreversível; é também um processo que nos afeta a todos na mesma medida e da mesma maneira". Em similaridade com Bauman (1999), sobre as concepções de globalização, Rajagopalan (2003, p. 25) pontua que estas transformações são advindas da "realidade marcada de forma acentuada por novos fenômenos e tendências irreversíveis como a globalização e a interação entre culturas".

Nessa perspectiva, no processo de globalização a articulação entre o global e o local “[...] não deve, naturalmente, ser confundido com velhas identidades, firmemente enraizadas em localidades bem delimitadas" (HALL, 2005, p. 78). Dito isso, por mais que a globalização trabalhe também a favor do capitalismo, é pouco provável que ela irá destruir ou suprimir as identidades nacionais. Talvez seja mais evidente que ela contribua para a construção de identificações outras, seja no âmbito local ou global. 
Desse modo, um dos motivos pelos quais as identidades estão em fase de transformações e renegociações é o contato entre diferentes sujeitos, com pensamentos heterogêneos e culturas distintas, propiciados pelo processo de globalização. Nesse sentido, com a construção da identidade cultural do professor de LI não é diferente, pois identidades são um processo de constructo contínuo, jamais estático. Devido a isso, com o processo de globalização, as identidades estão em constantes jogos identitários porque, a cada instante, surgem inúmeras identidades para serem testadas, conforme é postulado por Hall (2005, p. 12): "dentro de nós há identidades contraditórias, empurrando em diferentes direções, de tal modo que nossas identificações estão sendo continuamente deslocadas".

De fato, pensar a identidade do professor de LI em formação inicial, de forma isolada e centralizada, seria um pouco fantasiosa, uma vez que há multiplicidades de identidades culturais possíveis, e, portanto, passíveis de mudanças, visto estarem "em movimento" (BAUMAN, 2005) e serem "fluidas" (RAJAGOPALAN, 2003). Dessa forma, a identidade do profissional de língua inglesa em formação inicial encontra-se em processo contínuo de construção, pois as identidades "estão cada vez mais sendo percebidas como precárias e mutáveis, suscetíveis à renegociação constante" (RAJAGOPALAN, 2003, p. 69).

Nesse sentido, a construção da identidade cultural do professor de língua inglesa em formação, inserido no contexto local e global, sobre a cultura de ensinar uma língua estrangeira nos tempos de movências e transformações sócio-históricas, evocam os pensamentos de Hall, uma vez que, assim como ele, não concebemos a identidade:

como uma coisa acabada, deveríamos falar de identificação, e vêla como um processo em andamento. A identidade surge não tanto da plenitude da identidade que já está dentro de nós como indivíduos, mas de uma falta de inteireza que é "preenchida" a partir de nosso exterior, pelas formas através das quais nós imaginamos ser vistos por outros. (HALL, 2005, p. 39, grifos do autor)

Diante disso, o exercício da função de professor de língua inglesa, nos dias de hoje, implica pensar na construção de sua identidade a partir das transformações históricas e sociais que englobam, por exemplo, o compromisso social, bem como os valores, crenças sobre o que ensina e o que vive no ambiente escolar, dentre outras questões. Assim, estes 
aspectos fragmentam os sujeitos entre o eu e o outro, já que a profissão de professor é uma “profissão do conhecimento” (GARCIA, 2009, p. 8).

Após discutir, nesta seção, sobre a construção da identidade cultural do professor de língua inglesa em formação inicial, passemos, agora, para a seção seguinte, que aborda algumas visões sobre língua e cultura.

\section{Língua e Cultura}

Elucubrar sobre uma definição para o termo cultura não é uma tarefa nada fácil de realizar, ainda mais em um mundo globalizado. Conforme explica Lima (2009), estamos em um mundo cada vez mais interligado. Assim, conceituar 'cultura' torna-se complexo, visto que cultura por si mesma já é polissêmica, dessa forma, não devemos falar em cultura, mas, sim, em culturas.

A palavra cultura vem do latim colere e significa cultivar. Ela não é construída somente no meio familiar, mas também no meio social, pois somos sujeitos constituídos por linguagens nas mais diversas práticas e símbolos sociais. Nas palavras de Anastácio, Melo e Silva (2009, p. 24), o “embate entre natureza humana, que é inata, e a cultura, que é aprendida, interfere na estrutura da personalidade do indivíduo. Assim, a família é o primeiro contexto que transmite valores ao ser humano e o influencia na formação da sua identidade". Nesse ínterim, o conflito identitário é próprio dos indivíduos, uma vez que somos indivíduos cindidos nas nossas personalidades individuais que entram em confronto com as identidades adquiridas nos meios sociais, como na família, em que o ser humano adquire seus primeiros valores, bem como na profissão e, ainda, na escola, que tem a função de formar cidadãos para conviver em sociedade, de forma mais ampla.

Nessa perspectiva, tomamos emprestado da Antropologia o conceito de cultura que, na visão de Thompson (1995, p. 173), “a cultura [...] é o conjunto de crenças, costumes, ideias e valores, bem como os artefatos, objetos e instrumentos materiais que são adquiridos pelos indivíduos enquanto membros de um grupo ou sociedade”. Assim, nossas atitudes e existências estão impregnadas de ações culturais. Como aponta Tavares (2006), é por 
meio da cultura que permitimos nos conhecer e conhecer o outro e interpretamos o universo do qual fazemos parte.

Desse modo, as características culturais estão materializadas na nossa forma de ser e de nos apresentar para o mundo. Para Estermann (2010, p. 37-38), a cultura é "como nossa segunda pele, nos impregna em todos nossos aspectos de ser humano". Em outras palavras, ela - a cultura - está na nossa maneira de falar, no nosso modo de agir, nas nossas preferências alimentares, também nas nossas relações interpessoais e intrapessoais, nas nossas convicções religiosas e filosóficas etc.

Para Lima (2004), por sua vez, esclarece que é preciso levar em consideração as relações entre a língua e a cultura, pois, segundo ele, a língua tem o papel de ser não só transmissora de cultura, mas é por meio dela que nos expressamos e compartilhamos cultura. Desse modo, a língua está atravessada por questões ideológicas, políticas e sociais que constituem sujeitos mediante seus valores éticos e culturais situados historicamente. Assim, a palavra está sempre carregada de um conteúdo ou de um sentido ideológico ou vivencial (BAKHTIN, 2011).

Do mesmo modo, Ponzio (2008, p. 7) nos informa que "[...] um grupo social se reconhece como tal através de um processo complexo de diferenciação a respeito do que é diferente”. As questões culturais não são isoladas, elas aparecem e se constituem na interação dialógica, na qual cada sujeito cultural apresenta sua forma de ser na sua historicidade cultural e discursiva. Para Lima (2009, p. 180), "o papel da cultura [...] tem deixado de ser um assunto periférico para se tornar um tema de pesquisa e investigação no campo da linguística aplicada". Dado ao exposto, não se percebe o ensino de LI focado apenas nos quesitos gramaticais, na compreensão textual ou oral, mas evidencia-se a necessidade do aprendiz se situar no contexto social e cultural do idioma aprendido.

Em consonância com o que fora abordado, Agar (2002) postula que a cultura se encontra na língua, e que a língua carrega cultura. Logo, elas não estão desvinculadas, ainda que cada sociedade tenha consigo um recorte da sua representatividade histórica e social. 
Por este motivo, cada sujeito integrante de um grupo possui na sua subjetividade suas vivências e conhecimentos de mundo, adquiridos ao longo da história de cada um como sujeito sócio-histórico.

Passemos, agora, portanto, para seção seguinte, na qual discutirá sobre os aspectos metodológicos utilizados na pesquisa.

\section{Alguns passos metodológicos}

O escopo metodológico deste estudo é enunciado com base nos procedimentos técnicos do estudo de caso, pois, conforme Merriam (1998), Stake (1994) e Yin (2001), o método estudo de caso enquadra-se nos paradigmas qualitativos da pesquisa. Para Yin (2001, p. 69), “a metodologia pressupõe, em alguns casos, a existência de uma teoria prévia que será testada no decorrer da investigação e admite em outros casos a construção de uma teoria a partir dos achados da pesquisa”. Desse modo, esta investigação tem cunho qualitativo, além de ser motivada pelos estudos da Linguística Aplicada no Brasil. Nesse aspecto enunciativo, a seção metodológica revela-se extremamente importante, uma vez que é a parte de apresentação do corpus da pesquisa e, também, do método da pesquisa, os instrumentos de cotejamentos de dados, dentre outros.

A natureza desta pesquisa se enquadra nos paradigmas qualitativos com natureza etnográfica, haja vista, como afirma Siqueira (2008, p. 37) a “"pesquisa etnográfica' ou 'etnografia' tem se destacado como um dos métodos qualitativos mais importantes da pesquisa social". Nesse contexto, as enunciações de Flick (2004) nos direcionam para entender que os conhecimentos nas pesquisas qualitativas têm como objetivo estudar os saberes e as práticas dos sujeitos sociais que, de certa forma, pesquisador e participantes, se envolvam de maneira dialógica, reconstruindo suas identidades na interação recíproca.

Assim, "as pesquisas qualitativas consideram o contexto como algo que está intimamente ligado ao comportamento das pessoas na formação de suas experiências" (CANÇADO, 1994, p. 55). Logo, constatamos que o ser humano é constituído por um conjunto de valores e, também, visões de mundo sobre as coisas. Frente a isso, a pesquisa qualitativa 
"é uma atividade situada que localiza o observador no mundo que, por sua vez, é analisado a partir de um conjunto de práticas materiais e interpretativas" (DENZIN; LINCOLN, 2006, p. 17). Nesse contexto, essas práticas materiais e interpretativas revelam uma perspectiva dialógica, já que entendemos que o espaço de pesquisa "nos confronta com eventos de linguagem marcados pela interlocução" (FREITAS, 2002, p. 34).

De fato, as pesquisas qualitativas têm estreita ligação com o modo de vida da sociedade atual, ou seja, esse campo de pesquisas tem suas próprias características, que estão conectadas com o processo de transformação histórica e social. Desse modo, com o processo formativo do professor de língua inglesa em formação inicial não é diferente, pois ele faz parte da área do conhecimento que perpassa em diferentes épocas da história humana.

Nessa perspectiva, os procedimentos metodológicos são apreendidos como "uma sequência de operações que visam obter um resultado adequado às exigências da teoria" (FIORIN, 2002, p. 39). Nesse sentido, seguimos os pressupostos metodológicos fundamentados nas pesquisas qualitativa, associados a bases teóricas da Linguística Aplicada, a fim de gerar e cotejar os dados, a partir de entrevistas gravadas em áudio, bem como a partir de questionários, para, mais tarde, compreendermos o processo de construção da identidade dos sujeitos em questão.

Para tanto, os sujeitos da pesquisa são quatro estudantes, cursando o VIII semestre, no Curso de Graduação em Letras Língua Inglesa e Literaturas, em uma universidade situada no interior da Bahia. Por questões éticas, os nomes dos participantes deste trabalho não serão divulgados de forma real. No entanto, por escolha de cada participante, seguem suas nomenclaturas fictícias, a saber: Amélie, Henry, Laura e Sasuke Uchiha.

Uma vez exposta a metodologia e os sujeitos da pesquisa, passemos, a seguir, para seção de análises de dados, a fim de apresentar os resultados encontrados da pesquisa.

\section{A construção da identidade cultural do professor de LI em formação inicial: um estudo de caso}


Esta seção tem como finalidade apresentar as entrevistas feitas com base nos questionários, bem como as entrevistas gravadas em áudio. Dessa forma, os dados cotejados serão expostos, a seguir, com poucas supressões de fala dos sujeitos. As entrevistas gravadas serão transcritas com poucas marcas de oralidade apresentadas pelos respondentes da pesquisa. Vale lembrar que, neste estudo, só será apresentada uma pequena amostra da dissertação final de mestrado que deu origem a esse recorte aqui exposto. Para tanto, as questões expostas enfatizam aspectos culturais e identitários referentes ao processo de formação de professores de LI.

Com base nessas premissas, a primeira questão, apresentada aqui, esteve centrada no processo da construção da identidade do professor de LI ao adquirir formação em um idioma de língua estrangeira. Nesse sentido, buscamos saber dos sujeitos da pesquisa qual a opinião deles a respeito de aprender língua inglesa e seus aspectos culturais, levando em conta os questionamentos sobre se o aprendiz agregaria ou perderia valores na sua identidade ou na sua cultura ao aprender inglês. Diante disso, as respostas são postas em seguida:

Eu não acredito que ele perderia. Eu acredito que isso agregaria valor à sua identidade. No entanto, eu acredito que poderia perder se caso ela tomasse aquilo como parte da vida dele, renegando a própria cultura, por exemplo. Se ela aprende inglês, ela vai querer se comportar como um americano, por exemplo, e vai abominar a cultura que ela vivia antes, aí eu acho, nesse sentido, que a pessoa perderia a sua identidade, mas se ela não fizesse isso eu acho que agregaria sim. (Sasuke)

Eu penso que na situação de falante com certeza vai agregar valor, porque assim, o professor indivíduo de forma geral, ele não vai perder valores por estar ganhando outros. Penso que [...] vai somar porque você vai ter uma troca de conhecimentos, uma troca de informações de outra cultura, então é uma questão de relação [...] e na minha opinião, só tem a somar. (Laura)

Eu acredito que não perderia a identidade, porque o professor, ele tem que deixar o aluno ciente, né, que ele tá aprendendo ali uma nova língua, que ele vai aprender outra cultura, mas que ele não vai deixar sua cultura de lado, o seu idioma de lado. (Amélie) 
Com certeza, acredito que agregaria, porque a língua, ela está arraigada à cultura, não tem como você dissociar língua de cultura, elas se juntam, elas estão realmente interligadas, e o professor, a partir do momento, em que ele começa a ter, a adquirir informação nesse idioma ele passa tanto a compartilhar aspectos tanto da sua cultura como da cultura do outro, uma vez que você tem essa língua que estabelece comunicação entre diferentes culturas você pode crescer e agregar valor na sua formação de uma maneira geral, com certeza, tendo acesso a outros povos, a outras crenças e isso faz com que você cresça, tanto engaje você mesmo no discurso quanto os outros que é até mesmo o que os PCN falam sobre isso que a Língua Inglesa permite essas questões. (Henry)

De acordo com as explanações efetuadas pelos sujeitos da pesquisa, quando dialogamos com eles sobre o caso de perder ou agregar valores a sua identidade ou a sua cultura, ao aprender um novo idioma, constatamos que nas suas enunciações, de forma unânime, nenhum indivíduo perderia valor ao ter acesso a outros. Porém, conforme salientou Sasuke, a pessoa só perderia valores caso ela rejeitasse a sua cultura anterior no sentido de desvalorizá-la, imitando um falante nativo, como, por exemplo, um americano.

Os enunciados de Laura e Amélie estão similares, tendo em vista que a primeira, mesmo demonstrando insegurança na escolha do léxico para responder à questão, de forma geral, enfatiza que o indivíduo não irá perder valores ou sua identidade ao ganhar novos conhecimentos. Já a segunda, destaca a importância do professor nesse momento para deixar os alunos cientes de que eles não perderiam valores da sua cultura e nem da sua identidade ao estudarem uma língua estrangeira, pois os educandos não irão abandonar sua língua materna e nem sua cultura anterior. Vemos, portanto, nas informações colhidas através de Amélie, Laura e Sasuke similaridade com os pensamentos de Rajagopalan (2003, p. 69), quando este explica que: "quem aprende uma língua nova está se redefinindo como uma nova pessoa".

Na mesma perspectiva, Henry é mais enfático em sua resposta, ao postular que o aprendizado de um novo idioma agregaria valores à identidade do sujeito, pois, segundo ele, a língua está arraigada na cultura, portanto, não se desassocia uma da outra. Nesse sentido, o posicionamento dele encontra proximidade com o de Agar (2002), quando este 
afirma que a cultura se encontra na língua, e que a língua carrega cultura. Assim, para Henry, o professor só tem a ganhar em engajamento de conhecimentos culturais ao adquirir formação em um novo idioma, porque “identidade é um lugar de lutas e conflitos, é um espaço de construção de maneiras de ser e de estar na profissão” (NÓVOA, 2000, p. 34). Assim, o processo de construção da identidade do professor jamais ocorrerá de forma linear.

A segunda e última questão exposta neste recorte, busca saber dos sujeitos da pesquisa sobre a concepção que eles têm de cultura. Assim, as respostas são expostas a seguir:

Dentro da sala de aula eu acredito que cada pessoa traz consigo uma cultura diferente, então eu acredito que a cultura é algo particular, nunca é algo geral, específico, é diverso (Sasuke).

Bom, cultura para mim são aspectos próprios de um povo, aspectos que eu digo fica uma palavra muito ampla, porque são muitos aspectos, seja jeito de falar, jeito de se vestir, é jeito, jeito em todos os sentidos. [...] A forma como cumprimenta, a forma como a própria sociedade ela caminha é como se fosse... São realmente aspectos, não sei se teria outra palavra que eu entenderia como melhor colocar. Então, a forma de falar, por exemplo, língua, todas essas questões estão em volta desse conceito de cultura (Laura).

Bom, cultura é para mim um lugar... É um espaço onde... Cultura é onde você encontra é... Várias manifestações de um povo, de costumes. Acredito que é isso de diferentes costumes, né, entre diferentes povos (Amélie).

Bom, cultura é algo muito difícil de você conseguir dar uma definição para ela, né! Mas a cultura eu acho que seja um conjunto de as características gerais de todo um povo, né, de um lugar específico, de uma nação, de um país. Em todos os aspectos, acho que cultura está em parte no que a pessoa acredita, nos seus valores, envolve a questão, acho que a cultura envolve também a questão religiosa, muitas questões culturais são baseadas nisso. Enfim, tudo em que a pessoa acredita, na sua arte, no seu talento, acho que todos os aspectos de um povo em si, ela acaba sendo arregrada dentro da cultura de um povo em si, a vestimenta, a arte no geral (Henry). 
Diante das respostas dos sujeitos da pesquisa, constatamos a complexidade de definição para um termo tão polissêmico como cultura. Se, por um lado, Sasuke enfatiza que cultura é algo particular e nunca geral, ou seja, o posicionamento do respondente vai de encontro aos preceitos culturais que englobam tanto as características individuais quanto os arquétipos universais na polissemia de significados do termo cultura. Em outras palavras, ele não leva em consideração a "herança cultural" (LARAIA, 2005, p. 70) que é determinante no processo de subjetivação dos sujeitos, pois, desde o nascimento, o ser humano é inserido em um contexto cultural e nele são condicionados a seguir determinados valores sociais. É claro que, no decorrer do tempo, estes valores podem ser transformados, tendo em vista que são construções históricas, assim, são passíveis de mudanças.

Da mesma maneira, a cultura nos traz uma gama de significados ao mundo que nos rodeia, pois o homem se encontra em uma rede de unidades enunciativas históricas, com inserção no contexto de um mundo histórico e ideologicamente marcado, “[...] numa rede de relações intersubjetivas gestadas pela partilha de um sentido comum, que constitui seu mundo vivido, resultado das ações e dos pensamentos das gerações procedentes" (OLIVEIRA, 2005, p. 22). Nesse sentido, o homem está condicionado aos aspectos culturais de gerações anteriores, bem como em constante relação cultural com as coisas, com a natureza, ou seja, isso inclui a maneira como o ser humano interpreta o seu jeito de ser e agir na sua totalidade histórica e social.

Por outro lado, os retornos dados por Amélie, Laura e Henry se aproximam, ou seja, todas as respostas consideraram cultura um conjunto de diferentes costumes, aspectos ou manifestações. Amélie foi quem teve mais dificuldade em responder. Na sua resposta constam várias pausas longas, como se estivesse pensando na melhor opção de definição para o conceito de cultura. No entanto, Henry já deixa claro desde o início a complexidade de conceituação do termo, mas sua informação coincide em alguns pontos com as de Laura, já que ambos veem cultura como aspectos, manifestações populares que guiam a sociedade, como as artes e os movimentos artísticos, por exemplo. 
Nessa perspectiva, a partir das informações cotejadas dos sujeitos da pesquisa, podemos inferir que as respostas deles se aproximam do que Eagleton (2005, p. 54) também postula, haja vista que, para ele, "a cultura pode ser aproximadamente resumida como o complexo de valores, costumes, crenças e práticas que constituem o modo de vida de um grupo específico". Assim sendo, os sujeitos têm essas concepções culturais como um guia, pois, como é evidenciado no discurso de Laura, a cultura é a forma como a própria sociedade caminha, por meio dos seus costumes, representações e práticas sociais.

Nesse sentido, "A língua é um reflexo da cultura, pois, ao mesmo tempo em que a língua é uma parte da cultura, ela é também algo que a constitui" (MOREIRA; FIGUEIREDO, 2012, p. 147). Em outras palavras, "língua e cultura, mesmo diferentes entre si, formam um todo" (JIANG, 2002, p. 163). Em suma, tanto a língua quanto a cultura são partes constituintes da identidade do professor de LI que fazem uso da linguagem em um contexto social e simbólico.

Assim, ao finalizarmos a seção de análises, passemos, agora, para as Palavras Finais.

\section{Palavras Finais}

"A identidade é produto das sucessivas socializações" (DUBAR, 2005, p. 25).

A escolha dessa epígrafe para começar as palavras finais ocorreu porque parte do princípio que conduz este estudo, a identidade cultural do professor de LI, à luz dos pensamentos de pesquisadores da Linguística Aplicada, de estudiosos da identidade, em diálogo com autores de outros campos da linguagem que contribuíram para a construção deste trabalho, como, por exemplo, Bakhtin.

Este texto assumiu como objetivo geral analisar o processo de construção identitária e cultural de um grupo com quatro professores de LI. Para tanto, o presente estudo apresenta resultados a partir da problemática de como se dá a construção da identidade cultural do professor de língua inglesa em formação inicial. Nesta investigação, constatamos que a construção da identidade cultural do professor de língua inglesa em formação 
inicial se dá através das características da sociedade globalizada, pois, a partir do atual processo de globalização e, sobretudo, os dinamismos das interações culturais e identitárias subjetivam o processo formativo do professor de línguas contemporâneo.

De fato, os processos de construções identitárias estão em constantes transformações, o que Bauman (2005) denominou modernidade líquida, ou seja, estamos em tempos de dinamismo, de tempos fluídos. Diante disso, nada permanece imóvel, assim, com a construção da identidade do professor de língua inglesa em formação inicial não é diferente, pois, conforme ressalta Dubar (2005, p. 25), a identidade “[...] é construída na infância e, a partir de então, deve ser reconstruída no decorrer da vida. $\mathrm{O}$ indivíduo jamais a constrói sozinho: ele depende tanto dos juízos dos outros quanto de suas próprias orientações e autodefinições". Assim sendo, a identidade cultural do professor de LI é construída de forma contínua, nunca de forma isolada. Em outras palavras, a identidade do professor de LI se constrói através das relações com outras culturas, com outras identidades culturais, dentre outros aspectos.

Nessa perspectiva, vimos, com base nos dados cotejados dos participantes da pesquisa da pesquisa, que o professor de língua inglesa, ao adquirir formação nesse idioma, não perderia nem sua cultura e nem sua identidade. Muito pelo contrário, já que quem aprende um novo idioma e uma nova cultura só tem a acrescentar aspectos positivos, desde que seja de forma crítica/reflexiva.

Assim sendo, pelo que foi abordado, a construção da identidade cultural do professor de língua inglesa em formação inicial abre um leque de possibilidades de reflexão sobre os aspectos culturais e sociais da língua, tendo em vista que da voz e gestos dos professores de LI em formação inicial partem representações dos lugares que ocupam em uma instância social e historicamente constituída, já que, um profissional consciente do seu papel formador valoriza sua cultura e sua identidade, além disso, está sempre aberto para novas reflexões em busca de construção de novos saberes identitários e culturais de outros espaços de forma igualitária e respeitosa. 


\title{
THE CONSTRUCTION OF THE CULTURAL IDENTITY OF THE ENGLISH LAN- GUAGE TEACHER IN INITIAL TRAINING
}

\begin{abstract}
This study presents part of the results of the master's research - located in the Language and Education Research Line of the Postgraduate Program in Letters: Culture, Education and Languages -, which analyzed the construction of the English language teacher's identity (LI) in initial formation in times of interculturality. This work is affiliated to the studies of Applied Linguistics, in dialogue with voices such as those of Rajagopalan (2003), Lima (2009), among others. However, support is sought in authors from other areas, such as Bakhtin (2011), Estermann (2010), Hall (2005), Bauman (2005) and Thompson (1995). It's a case study, with an ethnographic nature, with the general objective of analyzing how the construction of the cultural identity of the English-speaking teacher, in initial formation, has been constructed within the university. Thus, the specific objectives are: to explore the constitution of the cultural identity of the language teacher in initial formation; to identify research possibilities that could contribute to reflect on the construction of the cultural identity of the LI teacher. The participants of the research are four teachers in initial formation in the Course of English Language Literature and Literature of a University located in Alto Sertão Baiano. Thus, based on the analysis of the interviews of the subjects of the research, the results found showed that the construction of the cultural identity of the teacher of LI in initial formation takes place through the characteristics of the globalized world.
\end{abstract}

KEYWORDS: Culture; Identity; Teacher Professional Development.

\section{REFERÊNCIAS}

AGAR, M. Language shock. 1. ed. Nova York: Perennial, 2002.

ANASTÁCIO, S. M. G; MELO, M. G. P. L.; SILVA, C. N. Nômades contemporâneos: famílias expatriadas e um mosaico de narrativas. Rio de Janeiro: Vieira \& Lent, 2009.

BAKHTIN, M. Problemas da poética em Dostoiévski. Trad. Paulo Bezerra. 3. Ed., Rio de Janeiro: Forense Universitária, 2002.

. O problema do texto na linguística, na filologia e em outras ciências humanas. In: BAKHTIN, M. Estética da criação verbal. Trad. Paulo Bezerra. 6. ed. São Paulo: WMF Martins Fontes, 2011.

BAUMAN, Z. Globalização: as consequências humanas. Rio de Janeiro: Jorge Zahar,1999. . Identidade: entrevista a Benedetto Vecchi. Tradução de Carlos Alberto Medeiros. Rio de Janeiro: Jorge Zahar, 2005.

BIZZO, N. Ciências: fácil ou difícil. $2^{\mathrm{a}}$ ed. 10ª impressão. São Paulo: Ed. Ática, 2002.

CANÇADO, M. Um estudo sobre a pesquisa etnográfica em sala de aula. In: Trabalhos em Linguística Aplicada. Campinas-SP, n. 23, p. 55-69, 1994. 
CRYSTAL, D. English as a Global Language. Cambridge: Cambridge University Press, 2003. DENZIN, N. K.; LINCOLN, Y. S. O planejamento das pesquisas qualitativa: teorias e abordagens. Porto Alegre: Artmed, 2006.

DUBAR, C. A socialização: construção das identidades sócias e profissionais. Trad. Andréa Stahel M. da Silva. São Paulo: Martins Fontes, 2005.

EAGLETON, T. A ideia de cultura. São Paulo: UNESP, 2005.

ESTERMANN, J. Interculturalidad: vivir la diversidade. La Paz: Enero, 2010.

FIORIN, J. L. Teoria e metodologia nos estudos discursivos de tradição francesa. In: SILVA, D; VIEIRA, J. (Org). Análise do discurso: percursos teóricos e metodológicos. Brasília: UnB. Oficina do Instituto de Letras: Plano, 2002. p. 39-74.

FLICK, U. Qualidade na pesquisa qualitativa. Coleção Pesquisa Qualitativa. Porto Alegre: Bookman, Artmed, 2009.

FREITAS, M. T. A abordagem sócio-histórica como orientadora da pesquisa qualitativa. Cadernos de pesquisa, São Paulo, n. 116, p. 21-39, jun. 2002.

GARCIA, M. C. Desenvolvimento profissional docente: passado e futuro. Sisito / Revista de ciências da educação, Lisboa, n. 8, p. 7-16, jan./abr. 2009.

HALL, S. A identidade cultural na pós-modernidade. 10. ed. Tradução de Tomaz Tadeu da Silva e Guacira Lopes Louro. Rio de Janeiro: DP\&A, 2005.

JIANG, W. The Relationship between culture and language. ELT Journal, v. 56, n. 3, 2002. Disponível em: <https://academic.oup.com/eltj/article-abstract/54/4/328/835293>. Acesso em: 15 fev. 2018.

LARAIA, R. de B. Cultura, um conceito antropológico. Rio de Janeiro: Jorge Zahar, 2005.

LIMA, D. C. de.(Org.) Foreign language learning and teaching: from theory to practice. Vitória da Conquista: Edições Uesb, 2004.

. Ensino e aprendizagem de língua inglesa: conversas com especialistas. São Paulo: Parábola Editorial, 2009.

MERRIAM, S. B. Qualitative research and case study applications in education. San Francisco: Jossey-Bass, 1998.

MOREIRA, T. A. S.; FIGUEREDO, C. J. A Importância do Componente Intercultural na Prática Docente de Línguas Estrangeiras. Gláuks, v. 12 n. 1. p. 147-168, 2012. Disponível em: <http://www.revistaglauks.ufv.br/arearestrita/arquivos_internos/artigos/Artigo_6_Thami_e_Carla_diagramado.pdf $>$. Acesso em: 19 fev. 2018.

NÓVOA, A. Os professores e as histórias da sua vida. In: NÓVOA, A. (Org.). Vidas de professores. 2. ed. Porto: Porto, 2000, p. 13-30. 
OLIVEIRA, M. A. Subjetividade e totalidade: Um confronto com as antropologias contemporâneas. Anais do Congresso Internacional de Filosofia "Pessoa e Sociedade: Perspectivas para o século XXI”. Braga: Universidade Católica de Portugal, 2005. p. 1-23.

PONZIO, A. A revolução bakhtiniana: o pensamento de Bakhtin e a ideologia contemporânea. São Paulo: Contexto, 2008.

RAJAGOPALAN, K. A. Por uma linguística crítica: linguagem, identidade e a questão ética: São Paulo: Parábola Editorial, 2003.

SIGNORINI, I. A questão da língua legítima na sociedade democrática um desafio para a linguística aplicada contemporânea. In: MOITA LOPES, L. P. da (Org.). Por uma linguística aplicada (in) disciplinar. São Paulo: Parábola Editorial, 2006, p. 169-190.

SIQUEIRA, D. S. Inglês como língua internacional: por uma pedagogia intercultural crítica. 2008. Tese (Doutorado em Letras e Linguística). Universidade Federal da Bahia, 2008.

STAKE, R. E. Case studies. In DENZIN, N. K.; LINCOLN, Y. S. Handbook of qualitative research. Thousand Oaks: Sage, 1994.

TAVARES, R. R. Conceitos de cultura no ensino/aprendizagem de LE. In: ROSEANNE, R. T. (Org.). Lingua, cultura e ensino. Maceió, Edufal, 2006.

THOMPSON, J. B. Ideologia e cultura moderna: teoria social na era dos meios de comunicação de massa. 5 ed. São Paulo, Vozes, 1995.

YIN, R. K. Estudo de caso - planejamento e métodos. 2. ed. Porto Alegre: Bookman, 2001.

Recebido em: 01/06/2019. Aprovado em: 17/07/2019. 